\title{
The Structure of Cube Tilings Under Symmetry Conditions
}

\author{
Andrzej P. Kisielewicz • Krzysztof Przesławski
}

Received: 18 November 2011 / Revised: 27 April 2012 / Accepted: 2 July 2012 /

Published online: 19 July 2012

(C) The Author(s) 2012. This article is published with open access at Springerlink.com

\begin{abstract}
Let $m_{1}, \ldots, m_{d}$ be positive integers, and let $G$ be a subgroup of $\mathbb{Z}^{d}$ such that $m_{1} \mathbb{Z} \times \cdots \times m_{d} \mathbb{Z} \subseteq G$. It is easily seen that if a unit cube tiling $[0,1)^{d}+t, t \in T$, of $\mathbb{R}^{d}$ is invariant under the action of $G$, then for every $t \in T$, the number $\mid T \cap(t+$ $\left.\mathbb{Z}^{d}\right) \cap\left[0, m_{1}\right) \times \cdots \times\left[0, m_{d}\right) \mid$ is divisible by $|G|$. We give sufficient conditions under which this number is divisible by a multiple of $|G|$. Moreover, a relation between this result and the Minkowski-Hajós theorem on lattice cube tilings is discussed.
\end{abstract}

Keywords Cube tiling

\section{Introduction}

An interest in cube tilings of $\mathbb{R}^{d}$ originated from the following question raised by Hermann Minkowski [29]: Characterize lattices $\Lambda \subset \mathbb{R}^{d}$ such that $[0,1)^{d}+\lambda, \lambda \in \Lambda$, is a cube tiling. Minkowski conjectured that such a lattice $\Lambda$ is of the form $A \mathbb{Z}^{d}$, where $A$ is a lower triangular matrix for which every entry on the main diagonal equals 1 . By a simple inductive argument it is equivalent to showing that $\Lambda$ contains an element of the standard basis. Geometrically, it means that the tiling $[0,1)^{d}+\lambda, \lambda \in \Lambda$, contains a column. This inspired Ott-Heinrich Keller [16, 17] to consider the problem of the existence of columns in arbitrary (nonlattice) cube tilings. Eventually, Minkowski's conjecture has been confirmed by Győrgy Hajós [11], while Jeffrey Lagarias and Peter Shor [24] constructed a cube tiling without columns in dimension 10. Geometric

A.P. Kisielewicz $\cdot$ K. Przesławski $(\bowtie)$

Wydział Matematyki, Informatyki i Ekonometrii, Uniwersytet Zielonogórski, ul. Z. Szafrana 4a, 65-516 Zielona Góra, Poland

e-mail: K.Przeslawski@wmie.uz.zgora.pl

A.P. Kisielewicz

e-mail: A.Kisielewicz@wmie.uz.zgora.pl 
and algebraic questions stemming from these problems attracted quite a number of researchers; see, e.g., [2, 3, 5, 6, 18, 20, 25-28, 30, 35].

Numerous authors pay their attention to tilings by clusters of cubes; see, e.g., [10, $12,13,21,31-34]$. Their investigations are rooted in coding theory and the GolombWelch conjecture [9]. These authors often deal with cubes which have their corners in $\mathbb{Z}^{d}$ and therefore the cluster tilings considered by them reduce to specific partitions of $\mathbb{Z}^{d}$. Other partitions of $\mathbb{Z}^{d}$ called disjoint covering systems are discussed in number theory; see, e.g., [1, 7].

A new stimulus to the theory of tilings came from Fuglede's conjecture [8]. A number of papers appeared at almost the same time where the set determining a cube tiling is characterized as follows: $[0,1)^{d}+t, t \in T$, is a cube tiling of $\mathbb{R}^{d}$ if and only if the system of functions $\exp (2 \pi \mathrm{i}\langle t, x\rangle), t \in T$, is an orthonormal basis of $L^{2}\left([0,1]^{d}\right)([14,15,21,23])$.

A reader who seeks an exposition concerning cube tilings is advised to consult $[22,33,36]$.

In this work we shall be concerned with cube tilings of $\mathbb{R}^{d}$ that are invariant under the action of certain groups of translations. Let $G \subset \mathbb{R}^{d}$ be a group. A cube tiling $[0,1)^{d}+t, t \in T$, is $G$-invariant if $T$ is $G$-invariant; that is, $t+x \in T$ whenever $t \in T$ and $x \in G$. Let us remark that if $T$ is a lattice, then the cube tiling is $T$ invariant. Observe that every $G$-invariant cube tiling leads to a tiling by clusters, where the union of all cubes $[0,1)+g, g \in G$, serves as a prototile.

Let $\boldsymbol{m}=\left(m_{1}, \ldots, m_{d}\right)$ be a vector whose coordinates are positive integers, and let $\boldsymbol{e}_{1}, \ldots, \boldsymbol{e}_{d}$ be the standard basis of $\mathbb{R}^{d}$. We say that the tiling $[0,1)^{d}+t, t \in T$, is $\boldsymbol{m}$-periodic if it is invariant under the action of the group generated by the vectors $m_{i} \boldsymbol{e}_{i}, i=1, \ldots, d$. (If $\boldsymbol{m}$ is unspecified, then we simply say that the tiling is periodic.) Our main result reads as follows.

Theorem 1 Let $\boldsymbol{m}$ be a vector whose coordinates are positive integers, and $G$ be a subgroup of $\mathbb{Z}^{d}$ such that $m_{1} \mathbb{Z} \times \cdots \times m_{d} \mathbb{Z} \subseteq G$. Let $[0,1)^{d}+t, t \in T$, be a $G$-invariant cube tiling of $\mathbb{R}^{d}$. Let $G^{i}=G \cap \mathbb{Z} \boldsymbol{e}_{i}$, and $\mu_{i}=\left|\mathbb{Z} / G^{i}\right|$. Then for every $t \in T$, the number $\left|T \cap\left(t+\mathbb{Z}^{d}\right) \cap\left[0, m_{1}\right) \times \cdots \times\left[0, m_{d}\right)\right|$ is divisible by $\operatorname{GCD}\left(\mu_{1}, \ldots, \mu_{d}\right)|G|$.

We found it convenient to express our theorem in terms of cube tilings of flat tori.

For a given $\boldsymbol{m}$, we define the (flat) torus $\mathbb{T}_{\boldsymbol{m}}^{d}$ as the set $\left[0, m_{1}\right) \times \cdots \times\left[0, m_{d}\right.$ ) with addition $\bmod \boldsymbol{m}$ :

$$
x \oplus y:=\left(\left(x_{1}+y_{1}\right) \bmod m_{1}, \ldots,\left(x_{d}+y_{d}\right) \bmod m_{d}\right) .
$$

Cubes in $\mathbb{T}_{m}^{d}$ are the sets of the form $[0,1)^{d} \oplus t$, where $t \in \mathbb{T}_{m}^{d}$. We say that $T \subset \mathbb{T}_{m}^{d}$ determines a cube tiling of $\mathbb{T}_{m}^{d}$ if the family $\mathcal{T}:=\left\{[0,1)^{d} \oplus t: t \in T\right\}$ is a tiling, that is, elements of $\mathcal{T}$ cover $\mathbb{T}_{\boldsymbol{m}}^{d}$ and are pairwise disjoint. Each $\boldsymbol{m}$-periodic cube tiling of $\mathbb{R}^{d}$ defines a cube tiling of $\mathbb{T}_{m}^{d}$ in an obvious manner. Those sets which determine cube tilings of $\mathbb{T}_{\boldsymbol{m}}^{d}$ are characterized as follows:

Lemma $2 T$ determines a cube tiling of $\mathbb{T}_{m}^{d}$ if and only if 
Fig. 1 The $G$-invariant cube tiling of $\mathbb{T}_{(2,2,2)}^{3}$ determined by $T=\{(0,0,0),(0,0,1),(1,1,0),(1,1,1)$, $(1,0,1 / 4),(1,0,5 / 4),(0,1,1 / 4),(0,1,5 / 4)\}$. The group $G$ consists of two elements, $(0,0,0)$ and $(1,1,1)$

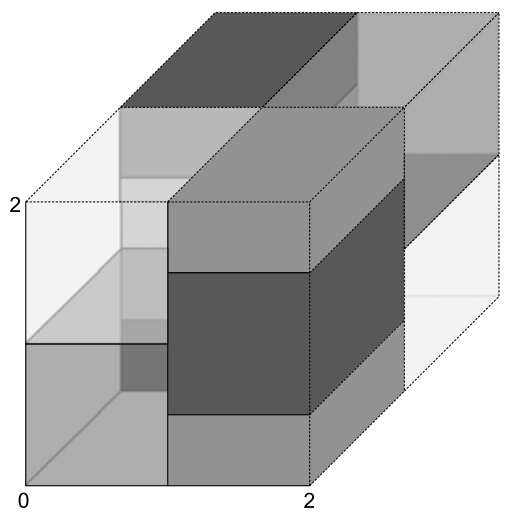

(1) $|T|=m_{1} \times \cdots \times m_{d}$,

(2) for every pair $x, y \in \mathbb{T}_{\boldsymbol{m}}$, there is $i \in[d]$ such that $x_{i}-y_{i}$ is a nonzero integer (Keller's condition).

(Compare [36, Lemma 8.3].)

Let us mention that the notion of $G$-invariance carries over to cube tilings of $\mathbb{T}_{\boldsymbol{m}}^{d}$. The integer lattice in $\mathbb{T}_{\boldsymbol{m}}^{d}$ is the subset $\mathbb{Z}_{\boldsymbol{m}}^{d}:=\mathbb{Z}_{m_{1}} \times \cdots \times \mathbb{Z}_{m_{d}}$ of $\mathbb{T}_{\boldsymbol{m}}^{d}$ consisting of all vectors with integer coordinates. If $T$ determines a cube tiling $\mathcal{T}$ of $\mathbb{T}_{\boldsymbol{m}}^{d}$ and $x \in T$, then the family $[0,1)^{d} \oplus t, t \in T \cap\left(x \oplus \mathbb{Z}_{m}^{d}\right)$, is said to be a simple component of the tiling $\mathcal{T}$. In light of Lemma 2 , we shall be mostly concerned with sets that determine cube tilings rather than cube tilings themselves. Therefore, we shall refer to $T \cap\left(x \oplus \mathbb{Z}_{\boldsymbol{m}}^{d}\right)$ as a simple component of $T$. For $u \in[0,1)^{d}$, let $C_{u}:=T \cap\left(u \oplus \mathbb{Z}_{\boldsymbol{m}}^{d}\right)$. It is clear that if $C_{u}$ is nonempty, then it is a simple component of $T$. Moreover, for each simple component $C$ of $T$, there is a unique element $u \in[0,1)^{d}$ such that $C=C_{u}$.

Theorem 1 can now be stated for the flat tori (Fig. 1).

Theorem 3 Let $T$ determine a cube tiling of $\mathbb{T}_{m}^{d}$. Let $G$ be a subgroup of $\mathbb{Z}_{m}^{d}$. Let $G^{i}=G \cap\left(\mathbb{Z}_{m_{i}} \boldsymbol{e}_{i}\right)$, where $\boldsymbol{e}_{i}$ is the ith element of the standard basis, and $\mu_{i}=m_{i} /\left|G^{i}\right|$. If $T$ is $G$-invariant, then each simple component of $T$ has its cardinality divisible by $\operatorname{GCD}\left(\mu_{1}, \ldots, \mu_{d}\right)|G|$.

\section{Proof of the Main Result}

We shall need the following:

Lemma 4 Let $V$ be a nonempty subset of $[0,1)^{d}$. If for every $v \in V$ and every $i \in[d]$, there is $y \in V$ such that $v_{q}=y_{q}$ whenever $q \neq i$ and $v_{i} \neq y_{i}$, then for every $v \in V$, there is $u \in V$ such that $v_{r} \neq u_{r}$ whenever $r \in[d]$. 
Proof By our assumptions, one can construct a sequence $v^{j}, j=0, \ldots, d$, consisting of elements of $V$ such that $v^{0}=v$ and $v_{q}^{j}=v_{q}^{j-1}$ for $q \neq j$ and $v_{j}^{j} \neq v_{j}^{j-1}$ for $j \in[d]$. Now, it suffices to set $u=v^{d}$.

Proof of Theorem 3 If the theorem were false, then there would exist a counterexample with minimal $d$. Clearly, $d>1$, as for $d=1$, the theorem is evidently true. Let $n=\operatorname{GCD}\left(\mu_{1}, \ldots, \mu_{d}\right)|G|$, and let $V \subset[0,1)^{d}$ consist of all $v$ such that $C_{v}$ is a simple component of $T$ and $\left|C_{v}\right|$ is not divisible by $n$. As a hypothetical counterexample is under discussion, $V$ is nonempty. We are going to show that $V$ satisfies the assumptions of Lemma 4 . We have to check only the case $i=d$, as for the remaining cases, the same reasoning applies. Let us define $s: \mathbb{T}_{\boldsymbol{m}}^{d} \rightarrow \mathbb{T}_{\boldsymbol{m}}^{d}$ by the formula $s(t)=\left(t_{1}, \ldots, t_{d-1},\left\lfloor t_{d}\right\rfloor\right)$. Let $S=s(T)$. By Lemma $2, s$ restricted to $T$ is a one-toone mapping, and $S$ determines a cube tiling of $\mathbb{T}_{m}^{d}$. Moreover, since $G$ is a subgroup of $\mathbb{Z}_{\boldsymbol{m}}^{d}$, it follows easily from the definition of $S$ that

$$
S \oplus G=\{s \oplus g: s \in S, g \in G\}=S .
$$

For $z \in[0,1)^{d}$, let $B_{z}=S \cap\left(z \oplus \mathbb{Z}_{m}^{d}\right)$. If $x \in T \cap[0,1)^{d}$, then $z=s(x) \in S \cap[0,1)^{d}$, and $B_{z}$ is a simple component of $S$. Let $\boldsymbol{m}^{\prime}=\left(m_{1}, \ldots, m_{d-1}\right)$. Then, by the definition of $S$, there are sets $S_{i} \subset \mathbb{T}_{\boldsymbol{m}^{\prime}}, i \in \mathbb{Z}_{m_{d}}$, such that

$$
S=S_{0} \times\{0\} \cup \cdots \cup S_{m_{d}-1} \times\left\{m_{d}-1\right\} .
$$

Since $S$ determines a cube tiling, each of the sets $S_{i}, i \in \mathbb{Z}_{m_{d}}$, determines a cube tiling of $\mathbb{T}_{\boldsymbol{m}^{\prime}}$. Let $G^{\prime}$ be the subgroup of $\mathbb{T}_{\boldsymbol{m}^{\prime}}$ defined by the equation $G^{\prime} \times\{0\}=\{g \in$ $\left.G: g_{d}=0\right\}$. By the definition of $S_{i}$ and the fact that $S \oplus G=S$ we have $S_{i} \oplus G^{\prime}=S_{i}$. Let $G^{\prime i}=G^{\prime} \cap\left(\mathbb{Z}_{m_{i}} \boldsymbol{e}_{i}\right)$ for $i \in[d-1]$. Clearly, $G^{\prime i} \cong G^{i}$. Let $z \in S \cap[0,1)^{d}$. The component $B_{z}$ decomposes in a similar way to $S$ :

$$
B_{z}=B_{0} \times\{0\} \cup \cdots \cup B_{m_{d}-1} \times\left\{m_{d}-1\right\} .
$$

If $B_{i}$ is nonempty, then it is a simple component of $S_{i}$. By induction, $\left|B_{i}\right|$ is divisible by $n^{\prime}=\operatorname{GCD}\left(\mu_{1}, \ldots, \mu_{d-1}\right)\left|G^{\prime}\right|$ for every $i \in \mathbb{Z}_{m_{d}}$. Let $H=\left\{g_{d}: g \in G\right\}$. Since $B_{z}$ is $G$-invariant, we have $\left|B_{i}\right|=\left|B_{j}\right|$ for every pair $i, j \in \mathbb{Z}_{m_{d}}$ such that $i-j \in H$. Therefore, $\left|B_{z}\right|$ is divisible by $n^{\prime}|H|=\operatorname{GCD}\left(\mu_{1}, \ldots, \mu_{d-1}\right)|G|$. A fortiori, $\left|B_{z}\right|$ is divisible by $n$. Let $z=s(v)$, where $v \in V$, and let $E_{v}=\left\{w \in[0,1)^{d} \cap T: s(w)=z\right\}$. Obviously $B_{z}$ is a disjoint union of the sets $s\left(C_{w}\right), w \in E_{v}$. Therefore, $\left|B_{z}\right|=$ $\sum_{w \in E_{v}}\left|C_{w}\right|$. Since $v \in E_{v}$ and the number $n$ divides $\left|B_{z}\right|$, and does not divide $\left|C_{v}\right|$, it follows that there is $y \in E_{v} \backslash\{v\}$ such that $n$ does not divide $\left|C_{y}\right|$ as well. Thus, $y$ belongs to $V$, and since $s(v)=s(y)$, we have $v_{q}=y_{q}$ whenever $q \neq i(=d)$. It means that $V$ satisfies the assumptions of Lemma 4 as expected. Consequently, there are two elements $v$ and $u$ in $V$ such that $v_{r} \neq u_{r}$ whenever $r \in[d]$. Let $t \in C_{v}$ and $t^{\prime} \in C_{u}$. Then $t_{j}-t_{j}^{\prime}$ is not an integer for any $j \in[d]$, which violates Keller's condition (Lemma 2). 


\section{Remarks}

A nonnegative integer $n$ is representable by $\boldsymbol{m} \in \mathbb{N}^{d}$ if there are nonnegative integers $n_{1}, \ldots, n_{d}$ such that

$$
n=n_{1} m_{1}+\cdots+n_{d} m_{d} .
$$

We have proved the following theorem [19], appealing to the result of Coopersmith and Steinberger on cyclotomic arrays [4]:

Theorem 5 If $T$ determines a cube tiling of a torus $\mathbb{T}_{m}^{d}$ and $C$ is a simple component of $T$, then $|C|$ is $\boldsymbol{m}$-representable.

It is a weakness of Theorem 3 that it is not a generalization of Theorem 5. The conjectural generalization should be the following:

Let $T$ determine a cube tiling of $\mathbb{T}_{m}^{d}$. Let $G$ be a subgroup of $\mathbb{Z}_{m}^{d}$. Let $G^{i}=$ $G \cap\left(\mathbb{Z}_{m_{i}} \boldsymbol{e}_{i}\right)$, where $\boldsymbol{e}_{i}$ is the ith element of the standard basis, and $\mu_{i}=m_{i} /\left|G^{i}\right|$. If $T$ is $G$-invariant, then each simple component of $T$ has the cardinality representable by $\left(\mu_{1}|G|, \ldots, \mu_{d}|G|\right)$.

Such a generalization would lead, for example, to a new proof of the MinkowskiHajós theorem on lattice cube tilings. We need less for this particular purpose. It would suffice to prove that, under the assumptions of Theorem 3, if all the numbers $\mu_{1}, \ldots, \mu_{d}$ are greater than 1 , then at least one of the simple components of $T$ has cardinality greater than $|G|$. Theorem 3 , as it is, suffices to show a restricted $p$-adic version of the Minkowski-Hajós theorem:

Let $\Lambda \subset \mathbb{R}^{d}$ be a cube tiling lattice. Suppose that there are a prime $p$ and a positive integer $k$ such that $p^{k} \boldsymbol{e}_{i} \in \Lambda$ for each $i \in[d]$. Then there is $j \in[d]$ such that $\boldsymbol{e}_{j} \in \Lambda$.

Indeed, let $T=\{\lambda \bmod \boldsymbol{m}: \lambda \in \Lambda\}$, where $\boldsymbol{m}=\left(p^{k}, \ldots, p^{k}\right)$. By the fact that $p^{k} \boldsymbol{e}_{i} \in \Lambda$ for $i \in[d]$, it follows that $T$ determines a cube tiling of $\mathbb{T}_{\boldsymbol{m}}^{d}$. Since $\Lambda$ is a subgroup of $\mathbb{R}^{d}, T$ is a subgroup of $\mathbb{T}_{\boldsymbol{m}}^{d}$. Let $G=\mathbb{Z}_{\boldsymbol{m}}^{d} \cap T$. Then $G$ is a nonempty subgroup of $T$, as $0 \in G$. Therefore, $T$ is $G$-invariant. By Theorem 3, the number $\left|\mathbb{Z}_{\boldsymbol{m}}^{d} \cap T\right|=|G|$ is divisible by $\operatorname{GCD}\left(\mu_{1}, \ldots, \mu_{d}\right)|G|$, which, by taking into account that all $\mu_{i}$ are powers of $p$, readily implies that there is $j$ such that $\mu_{j}=1$. Thus, $p^{k}=\left|G^{j}\right|$, and consequently, $\boldsymbol{e}_{j} \in G^{j} \subseteq T$.

Open Access This article is distributed under the terms of the Creative Commons Attribution License which permits any use, distribution, and reproduction in any medium, provided the original author(s) and the source are credited.

\section{References}

1. Berger, M.A., Felzenbaum, A., Fraenkel, A.: A non-analytic proof of the Newman-Znám result for disjoint covering systems. Combinatorica 6, 235-243 (1986)

2. Corrádi, K., Szabó, S.: Cube tiling and covering a complete graph. Discrete Math. 85, 319-321 (1990)

3. Corrádi, K., Szabó, S.: A combinatorial approach for Keller's conjecture. Period. Math. Hung. 21, 95-100 (1990)

4. Coppersmith, D., Steinberger, J.: On the entry sum of cyclotomic arrays. Integers 6, A28 (2006)

5. Dutour Sikirić, M., Itoh, Y.: Combinatorial cube packings in the cube and the torus. Eur. J. Comb. 31, 517-534 (2010) 
6. Dutour Sikirić, M., Itoh, Y., Poyarkov, A.: Cube packings, second moment and holes. Eur. J. Comb. 28, 715-725 (2007)

7. Feldman, D., Propp, J., Robins, S.: Tiling lattices with sublattices, I. Discrete Comput. Geom. 46, 184-186 (2011)

8. Fuglede, B.: Commuting self-adjoint partial differential operators and a group theoretic problem. J. Funct. Anal. 16, 101-121 (1974)

9. Golomb, S., Welch, L.: Perfect codes in the Lee metric and the packing of polyominoes. SIAM J. Appl. Math. 18, 302-317 (1970)

10. Gravier, S., Mollard, M., Payan, Ch.: On the non-existence of 3-dimensional tiling in the Lee metric. Eur. J. Comb. 19, 567-572 (1998)

11. Hajós, G.: Über einfache und mehrfache Bedeckung des $n$-dimensionalen Raumes mit einem Würfelgitter. Math. Z. 47, 427-467 (1941)

12. Horak, P., Al Bdaiwi, B.: Non-periodic tilings of $\mathbb{R}^{n}$ by crosses. Discrete Comput. Geom. 47, 1-16 (2012)

13. Horak, P.: Tilings in Lee metric. Eur. J. Comb. 30, 480-489 (2009)

14. Iosevich, A., Pedersen, S.: Spectral and tiling properties of the unit cube. Int. Math. Res. Not. 16, 819-828 (1998)

15. Jorgensen, P., Pedersen, S.: Spectral pairs in Cartesian coordinates. J. Fourier Anal. Appl. 5, 285-302 (1999)

16. Keller, O.H.: Über die lückenlose Erfüllung des Raumes Würfeln. J. Reine Angew. Math. 163, 231248 (1930)

17. Keller, O.H.: Ein Satz über die lückenlose Erfüllung des 5- und 6-dimensionalen Raumes mit Würfeln. J. Reine Angew. Math. 177, 61-64 (1937)

18. Kisielewicz, A.P., Przesławski, K.: Polyboxes, cube tilings and rigidity. Discrete Comput. Geom. 40, 1-30 (2008)

19. Kisielewicz, A.P., Przesławski, K.: The coin exchange problem and the structure of cube tilings. Electron. J. Combin. 19(2), P26 (2012)

20. Kolountzakis, M.N.: Lattice tilings by cubes: whole, notched and extended. Electron. J. Comb. 5, R14, 11 (1998)

21. Kolountzakis, M.N.: Packing, tiling, orthogonality and completeness. Bull. Lond. Math. Soc. 32, 589599 (2000)

22. Kolountzakis, M.N.: The study of translational tiling with Fourier analysis. In: Fourier Analysis and Convexity. Appl. Numer. Harmon. Anal, pp. 131-187. Birkhäuser, Boston (2004)

23. Lagarias, J.C., Reeds, J.A., Wang, Y.: Orthonormal bases of exponentials for the $n$-cube. Duke Math. J. 103, 25-37 (2000)

24. Lagarias, J.C., Shor, P.W.: Keller's cube-tiling conjecture is false in high dimensions. Bull. Am. Math. Soc. 27, 279-287 (1992)

25. Lagarias, J.C., Shor, P.W.: Cube tilings and nonlinear codes. Discrete Comput. Geom. 11, 359-391 (1994)

26. Łysakowska, M., Przesławski, K.: On the structure of cube tilings and unextendible systems of cubes in low dimensions. Eur. J. Comb. 32, 1417-1427 (2011)

27. Łysakowska, M., Przesławski, K.: Keller's conjecture on the existence of columns in cube tilings of $\mathbb{R}^{n}$. Adv. Geom. 12, 329-352 (2012)

28. Mackey, J.: A cube tiling of dimension eight with no facesharing. Discrete Comput. Geom. 28, 275279 (2002)

29. Minkowski, H.: Diophantische Approximationen. Teubner, Leipzig (1907)

30. Perron, O.: Über lückenlose Ausfüllung des $n$-dimensionalen Raumes durch kongruente Würfel. Math. Z. 46, 1-26 (1940)

31. Schwartz, M.: Quasi-cross lattice tilings with applications to flash memory. IEEE Trans. Inf. Theory 58, 2397-2405 (2012)

32. Stein, S.K.: Tiling, packing, and covering by clusters. Rocky Mt. J. Math. 16, 277-321 (1986)

33. Stein, S.K., Szabó, S.: Algebra and Tiling: Homomorphisms in the Service of Geometry. American Mathematical Association, Washington (1994)

34. Špacapan, S.: Non-existence of face-to-face four dimensional tiling in the Lee metric. Eur. J. Comb. 28, 127-133 (2007)

35. Szabó, S.: Cube tilings as contributions of algebra to geometry. Beiträge Algebra Geom. 34, 63-75 (1993)

36. Zong, C.: The Cube: A Window to Convex and Discrete Geometry. Cambridge Tracts in Mathematics, vol. 168. Cambridge University Press, Cambridge (2006) 